\title{
Minimally invasive Ivor Lewis esophagectomy in 10 steps
}

\footnotetext{
From the Thoracic Service, Department of Surgery, Memorial Sloan Kettering Cancer Center, New York, NY. This study was supported, in part, by the National Institutes of Health/National Cancer Institute Cancer Support Grant P30 CA008748.

Disclosures: D.M. serves as a consultant for Johnson \& Johnson, Urogen, and Boston Scientific. C.H. reported no conflicts of interest.

The Journal policy requires editors and reviewers to disclose conflicts of interest and to decline handling or reviewing manuscripts for which they may have a conflict of interest. The editors and reviewers of this article have no conflicts of interest.

Received for publication Dec 13, 2020; revisions received April 6, 2021; accepted for publication April 23, 2021; available ahead of print Aug 8, 2021.

Address for reprints: Daniela Molena, MD, Thoracic Service, Department of Surgery, Memorial Sloan Kettering Cancer Center, 1275 York Ave, New York, NY 10065 (E-mail: molenad@mskcc.org).

JTCVS Techniques 2021;10:489-94

2666-2507

Copyright $(2021$ The Author(s). Published by Elsevier Inc. on behalf of The American Association for Thoracic Surgery. This is an open access article under the CC BY-NC-ND license (http://creativecommons.org/licenses/bync-nd/4.0/).

https://doi.org/10.1016/j.xjtc.2021.04.038
}

Video clip is available online.

Feature Editor's Introduction-It is reasonable to submit that esophagectomy is one of the most complex, unforgiving procedures in surgery. Recent analyses of the National Cancer Database have demonstrated that the number of minimally invasive esophagectomies performed in the United States had surpassed the number of open esophagectomies by 2015. This should be considered as a trailblazing accomplishment by early pioneers in our field that drove this innovation and as a testament to the perseverance and skill of contemporary thoracic surgeons who treat patients with cancer and other ailments of the esophagus. Moreover, our surgeons stood up to investigate this new, potentially high-risk procedure scientifically. Two multicenter randomized trials, TIME (Traditional Invasive Vs Minimally Invasive Esophagectomy) and MIRO (Open Versus Laparoscopically Assisted Esophagectomy for Cancer: A Multicentric Phase III Prospective Randomized Controlled Trial), were reported in the last decade and demonstrated that minimally invasive approaches to esophagectomy were associated with decreased surgical morbidity when compared with open esophagectomy without sacrificing oncologic outcomes, including extent of nodal dissection, $R O$ resection status, and disease-free survival. In the Video Atlas article that follows, the authors distill an intricate, bicavitary minimally invasive operation into the 10 dominant steps that make up the laparoscopic and thoracoscopic Ivor Lewis esophagectomy.

\section{METHODS} videos and images.

a. Assistant port resident

a. Surgeon port

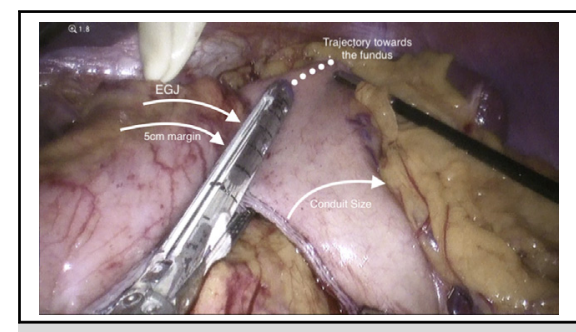

Creation of the gastric conduit.

CENTRAL MESSAGE

This article is a video atlas that describes the steps of a minimally invasive Ivor Lewis esophagectomy.

See Commentary on page 495.

Minimally invasive esophagectomy is the preferred approach for surgical resection of the esophagus in many centers, allowing for significant reduction in the morbidity associated with open resection ${ }^{1,2}$ while offering equivalent oncological outcomes..$^{3-5}$ Our approach to minimally invasive Ivor Lewis esophagectomy will be described in this chapter in 10 steps.

Patients gave informed consent for the capture and use of operative

\section{Trocar Placement_Abdomen (Figure 1)}

1. 10-mm optical trocar for entry-left costal margin at midclavicular line

b. Used to staple left gastric artery/vein

2. 5- or 10-mm trocar for camera-midline below falciform ligament

a. Camera holder can be used if there is no physician assistant/student/

3. 2 additional 5-mm trocars—right and left upper quadrants

a. Surgeon port-right side

b. Assistant port-left side

4. 10-mm trocar-right flank

b. Used for tubularization of the stomach

5. Nathanson liver retractor-below xiphoid process

Bryan M. Burt, MD 


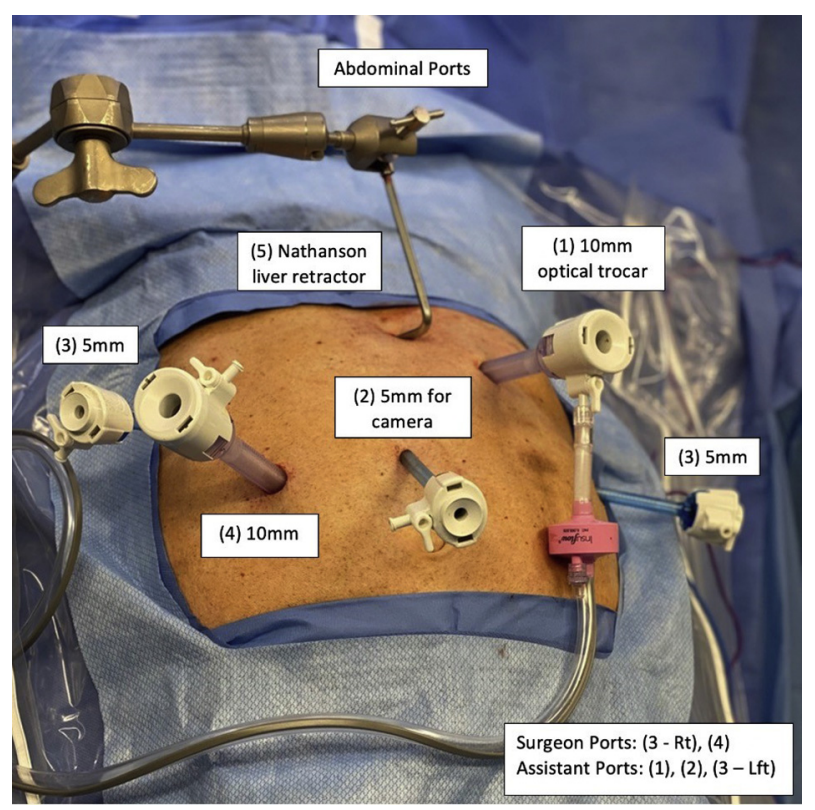

FIGURE 1. Trocar placement-abdomen.

\section{Port Placement-Chest (Figure 2)}

1. 10-mm port-posterior axillary line in seventh intercostal space (will be replaced by wound protector)
a. Surgeon port
b. Used to staple the azygous vein
c. Used to staple the stomach when the conduit is divided from the specimen
d. End-to-end anastomosis (EEA) goes through the wound protector

2. 5-mm camera port-posteriorly to 10 -mm port in ninth intercostal space

a. The camera is moved here from trocar \#1 after entry and insufflation 3. 10-mm port-midaxillary line in the third or fourth intercostal space

\section{a. Surgeon port}

b. Used to staple the esophagus

4. 5-mm port—seventh intercostal space between spine and scapula

a. Assistant port

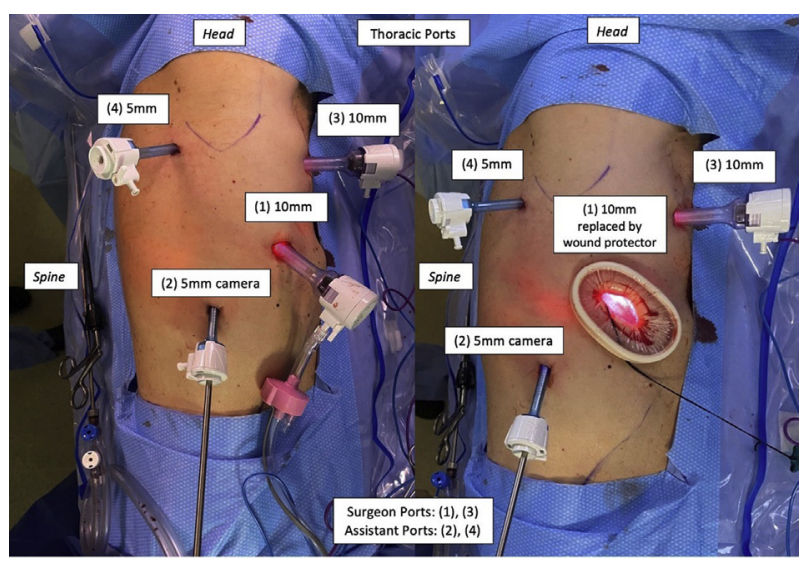

FIGURE 2. Port placement—chest.

\section{Instruments}

1. OrVil 25-mm 4.8-mm stapler

2. Endovascular staplers

3. Carter-Thomason fascial closure device

4. Thoracoscopic specimen bag

5. LigaSure

6. Endo Stitch

\section{RESULTS}

\section{Abdomen}

Lymphadenectomy of the celiac trunk and division of the left gastric pedicle. After the patient is placed in a supine position with arms out and feet against the padded foot board, they are prepped, draped, and undergo appropriate trocar placement. The peritoneal cavity is examined to rule out unresectable or metastatic disease. The gastrohepatic ligament is entered and divided until dissection reaches the right crus, and then moves inferiorly to expose the celiac trunk. The left gastric, splenic, and common hepatic arteries are visualized, and their associated lymph nodes are dissected to perform a complete lymphadenectomy. The left gastric pedicle is dissected starting along the superior edge of the pancreas and continues along the hepatic artery, to the right crus and behind the left gastric artery. The left gastric vein is clipped and divided with shears, and the artery is stapled at its origin. These are taken separately so that a complete lymphadenectomy can be performed without compromise. Dissection then continues along the splenic artery and the lateral aspect of the left crus until the first short gastric vessels running along the left crus are identified and divided (Video 1).

Mobilization of the greater curvature. The stomach is retracted anteriorly and towards the liver to expose the gastrocolic ligament, which is divided 3 to $4 \mathrm{~cm}$ away from the right gastroepiploic artery. This artery will serve as the main blood supply of the gastric conduit and should be carefully spared and yet completely mobilized to its base. Once the lesser sac is entered, dissection continues along the greater curvature of the stomach toward the fundus through

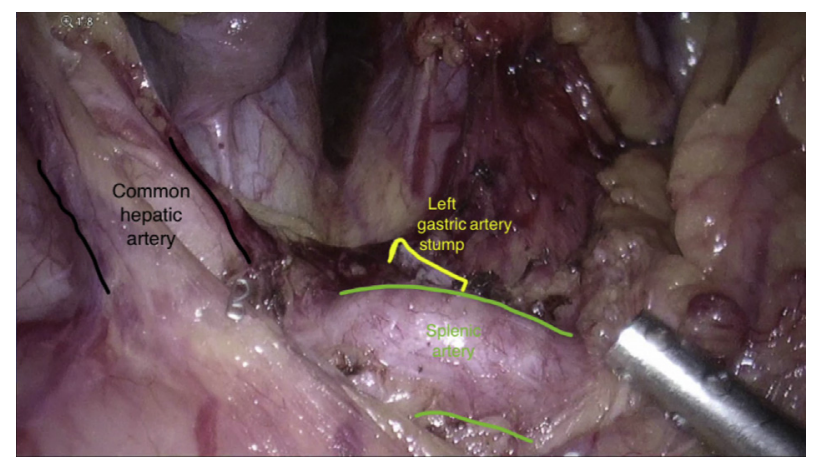

VIDEO 1. Step 1. Video available at: https://www.jtcvs.org/article/ S2666-2507(21)00515-0/fulltext. 


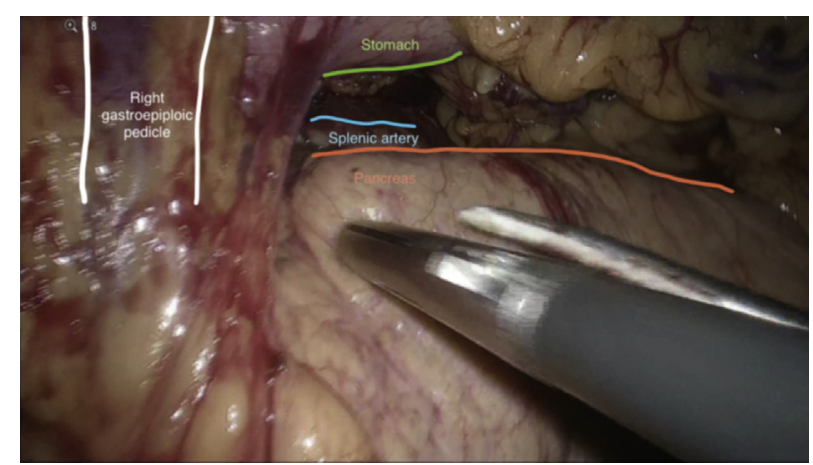

VIDEO 2. Step 2. Video available at: https://www.jtcvs.org/article/ S2666-2507(21)00515-0/fulltext.

the gastrosplenic ligament until it meets the dissection of the left crus, preserving the greater curvature fat. This is performed carefully so that the short gastric vessels are taken with a long stump on the splenic side and so that the spleen itself is not injured. It is usually easier to divide the most posterior short gastric vessels coming from the retroperitoneum along the splenic artery, as mentioned in step 1 , so that the most proximal attachments between the stomach and spleen can be approached from both sides of the splenic hilum. The gastrocolic ligament is then divided distally, making sure to identify the plane between the transverse mesocolon and the gastroepiploic pedicle because they are often fused at the level of the middle colic vessels. After separating the transverse mesocolon from the gastrocolic ligament, the gastrocolic ligament is divided all the way to the duodenum. The base of the right gastroepiploic artery is dissected to the inferior edge of the pancreas, and the posterior attachments between the pancreas and stomach are completely divided. Adhesions from a prior cholecystectomy should also be taken. At this point, the pylorus should be able to reach the hiatus (Video 2).

Transhiatal dissection. The esophagus is circumferentially dissected at the level of the hiatus, allowing for passage of a Penrose drain. This will serve as a handle to aid in retraction. The plane of dissection will be carried from pleura to pleura, pericardium to aorta, to the level of the inferior pulmonary vein. Periesophageal lymph nodes are kept with the specimen. Depending on tumor extension, hiatal structures (diaphragm, pericardium, and pleura) can be resected en bloc with the esophagus. It is important to remove the paracardial nodes located between the esophagus and the pericardium (Video 3).

Creation of the conduit. The orogastric tube is removed to prevent stapling across it as tubularization of the stomach is performed. A 4- to 5-cm wide gastric conduit is created using a series of surgical staplers, starting just proximal to the pylorus on the lesser curvature and continuing toward the fundus. In the majority of cases, the starting distance proximal to the pylorus is about $5 \mathrm{~cm}$, but we tailor this step to

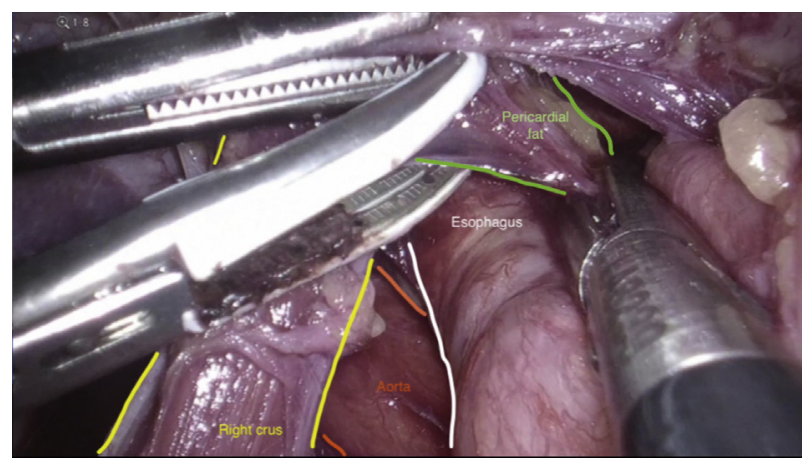

VIDEO 3. Step 3. Video available at: https://www.jtcvs.org/article/ S2666-2507(21)00515-0/fulltext.

the patient's anatomy and tumor location, considering the distal margin and ability to bring the conduit to either the chest or neck. Moving closer to the pylorus will allow for a longer conduit. It is important to stretch and straighten the stomach from the fundus to the pylorus while stapling the conduit to avoid spiraling of the staple line. Appropriate distance from the esophagogastric junction is maintained to preserve adequate distal margin while moving toward the fundus. The last 3 to $5 \mathrm{~cm}$ of the gastric conduit can be left attached to the specimen, which allows for easy retrieval into the chest. If a tumor is bulky, the stomach should be divided completely and secured to the lesser curvature instead, which will allow for easier transposition in the chest. No pyloric drainage procedure is necessary. ${ }^{6}$ If a hiatal hernia is found, the diaphragmatic hiatus should be closed with one or two stitches to help prevent paraconduit herniation. Securing the conduit to the right crus from the chest does not decrease the occurrence of paraconduit herniation in our experience (Video 4).

Feeding tube placement. The transverse mesocolon is lifted superiorly to expose the ligament of Treitz. A mobile portion of proximal jejunum is grasped and elevated to the left abdominal wall. A diamond pattern surrounding the intended jejunostomy site is created with 4 absorbable

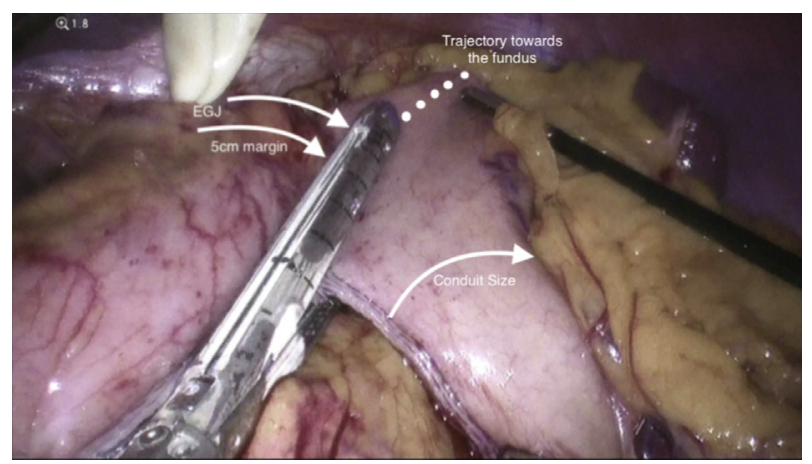

VIDEO 4. Step 4. Video available at: https://www.jtcvs.org/article/ S2666-2507(21)00515-0/fulltext. 
sutures, which are brought through the abdominal wall using a Carter-Thomason fascial closure device (CooperSurgical, Trumbull, Conn). A percutaneous jejunostomy is created using a Seldinger technique. The 4 anchoring sutures are tied externally within the subcutaneous layer of the abdominal wall. The tube is advanced into the bowel lumen and distal jejunum. An additional stitch is placed distally to the insertion site to avoid torsion of the jejunal loop (Video 5).

\section{Thoracic Phase}

Opening of the anterior and posterior pleura and division of the azygous vein. After the patient is placed in a left lateral decubitus position with table flexed at the hip, the appropriate ports are placed as described in our methods. The inferior pulmonary ligament is then divided to the level of the inferior pulmonary vein, and its associated lymph nodes are resected. The mediastinal pleura is dissected anteriorly between the esophagus and the lung up to the level of the azygous vein, and then the vein is divided using a vascular load stapler. This dissection is then carried posteriorly between the esophagus and the aorta and inferiorly to the diaphragm, until it meets the transhiatal dissection performed during the abdominal phase. The pleura above the azygos vein is preserved and will be used to suspend the gastric conduit at the end of the procedure (Video 6).

Circumferential mobilization of the esophagus, identification of airways, and division of the esophagus. The dissection starts at the level of the right inferior pulmonary vein, where it is usually easy to find the pericardial plane. The dissection is then taken inferiorly along the inferior vena cava to the diaphragmatic crus. The Penrose drain that was placed during the transhiatal dissection is visualized and can be used to retract the esophagus and expose planes of dissection of the esophagus from the mediastinum. Once the esophagus distal to the inferior pulmonary vein is circumferentially dissected from the pericardium, aorta, and left pleura, the dissection proceeds anteriorly

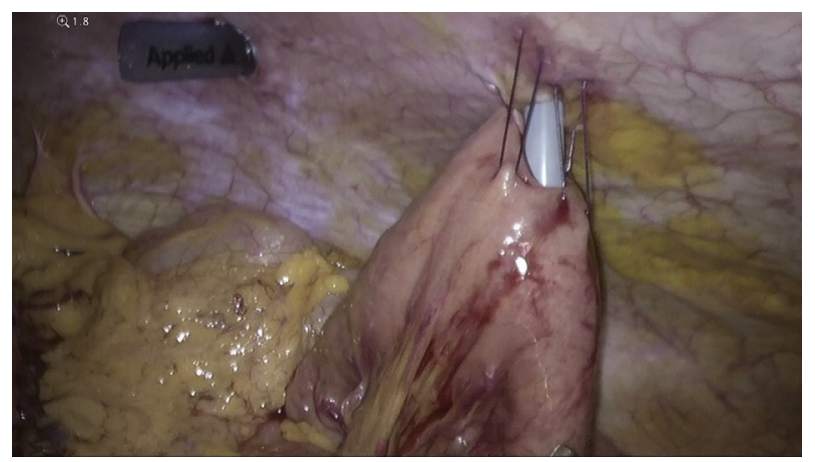

VIDEO 5. Step 5. Video available at: https://www.jtcvs.org/article/ S2666-2507(21)00515-0/fulltext.

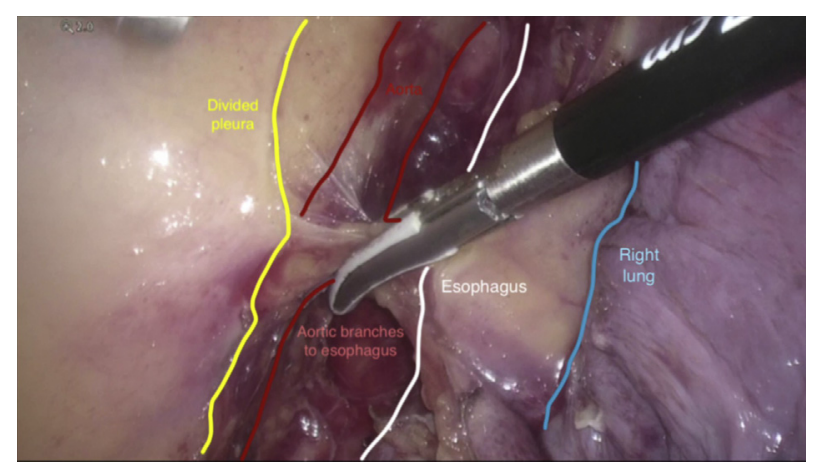

VIDEO 6. Step 6. Video available at: https://www.jtcvs.org/article/ S2666-2507(21)00515-0/fulltext.

along the main branch of the anterior vagus nerve. By retracting on the nerve, the plane between the esophagus and the bronchus intermedius is identified, and this plane is dissected superiorly over the trachea and posteriorly toward the left main bronchus. It is easier to identify the full extension of the airways if the infracarinal nodes are left behind to dissect separately after division of the esophagus. If en bloc dissection is required by tumor extension, visualization of the takeoff of the left main bronchus from the trachea will help avoid injury to the distal left main bronchus sitting behind the infracarinal nodes. Careful attention should be spent in the area delineated by the left inferior pulmonary vein and the distal left main bronchus as the presence of lymph nodes obscures the course of these structures and injury in this area could be catastrophic. After the airways are dissected from the esophagus, posterior dissection from the left pleura, aorta, and aortic arch is easily performed. As this dissection occurs it is important to carefully identify lymphatic branches from the thoracic duct and arterial branches from the aorta. For branches that are large in size or short, clips can be used as a failsafe. The esophagus is divided with linear staplers just above the azygos vein or higher if required by tumor margins. The tips of the stapler should be visualized before division to avoid injury to surrounding structures. The esophageal stump is mobilized about $2 \mathrm{~cm}$ away from the pleura, the trachea, and lateral mediastinal attachments to allow free movement of the OrVil within the stump (Video 7).

Gastric conduit pullup, division of the specimen, pathological assessment of margin, and mediastinal lymphadenectomy. The conduit is pulled up into the chest enough to divide the specimen, with careful attention to maintain the orientation and avoid twisting. The staple line should remain oriented to the patient's right. The specimen is divided from the gastric conduit using a linear stapler, making sure to preserve the distal margin from the gastroesophageal junction at the fundus. The incision at the seventh intercostal space is enlarged to 4 to $5 \mathrm{~cm}$, and 


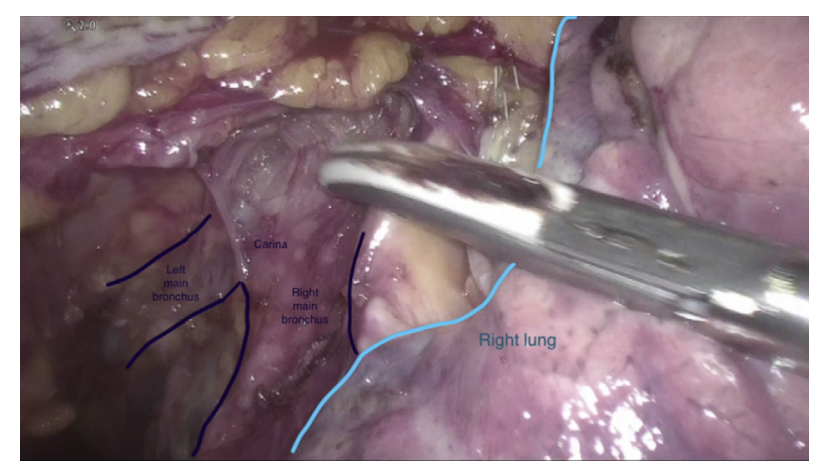

VIDEO 7. Step 7. Video available at: https://www.jtcvs.org/article/ S2666-2507(21)00515-0/fulltext.

insufflation is stopped. A wound protector is placed in the intercostal space, and the specimen is then removed using a bag and sent for pathologic assessment. While awaiting results of the frozen section, a complete subcarinal lymph node dissection is performed. We start at the corner between the right inferior pulmonary vein and the bronchus intermedius, proceed toward the carina along the bronchus intermedius, identify the left main bronchus, and then dissect the nodes at the carina, approaching from both the right and left main bronchi, as these nodes often extend anteriorly and superiorly to the carina. The packet is then dissected from the left main bronchus and the left inferior pulmonary vein. A more extensive right and/or left paratracheal lymphadenectomy is only performed for esophageal squamous cancers or in case of tumor involvement of these nodes before and/or after treatment (Video 8).

Passage of the OrVil and assessment of conduit perfusion. The anesthesiologist advances the oral anvil for the OrVil so that the tip of the tubing reaches the proximal staple line. The esophageal stump should be stabilized with graspers on both sides, and cutting is utilized to create an opening in the posterior right side of the staple line where the anvil is passed through. The opening for the OrVil should be just next to the staple line of the esophageal stump

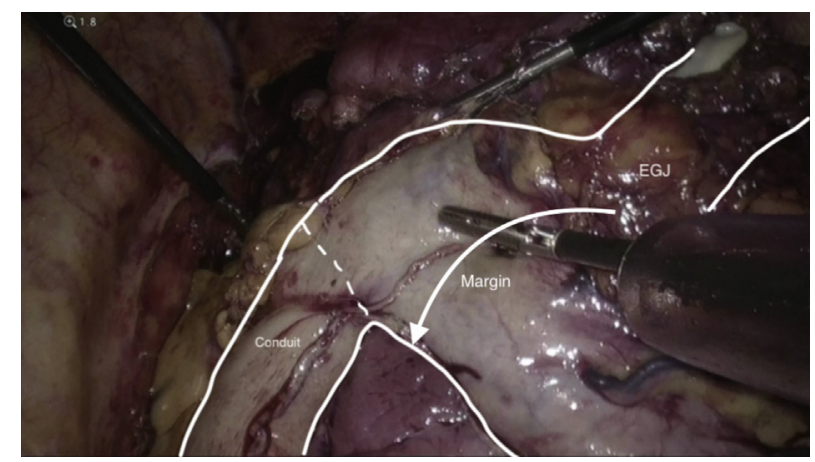

VIDEO 8. Step 8. Video available at: https://www.jtcvs.org/article/ S2666-2507(21)00515-0/fulltext.

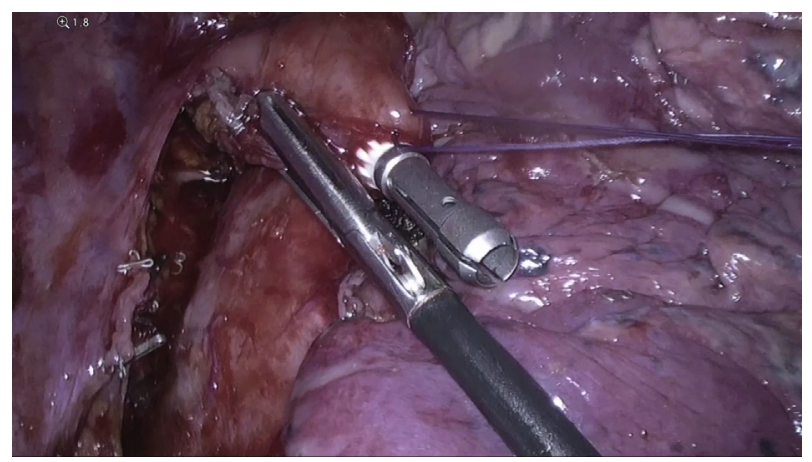

VIDEO 9. Step 9. Video available at: https://www.jtcvs.org/article/ S2666-2507(21)00515-0/fulltext.

to avoid leaving devascularized tissue at the anastomotic site. We prefer to pass the OrVil at the right corner of the staple line so that there is only one point of crossing between lineal and circular staple lines where a potential area of ischemia can lead to leakage (Video 9). The conduit is then pulled up completely into the chest, and perfusion is assessed with indocyanine green and fluorescent camera. If an area of poor perfusion is noted, this area should be avoided when making the anastomosis and resected after the anastomosis is completed.

Anastomosis. Once the proximal and distal margins are determined by pathology to be negative for disease, the anastomosis is created. This is the most important part of the case as perioperative morbidity is mostly driven by anastomotic healing. The proximal conduit staple line is opened so that an EEA circular stapler can be inserted. Tension between the conduit and the EEA will facilitate advancement of the pin through the stomach wall. The pin should come out next to the greater curvature toward the lung side so that the greater curvature's omentum can be interposed between the airways and the anastomosis. We use a right-angle grasper to pull the omentum toward the mediastinum while we advance the pin through the stomach. An OrVil grasper is used to engage the anvil with the EEA. Since the pin will push the OrVil to tilt perpendicularly, it might take a while for the anvil to clasp the pin of the EEA. It is important not to force this step, otherwise the anvil may become loose and unable to clasp around the pin. Once the anastomosis is completed, the donuts should be inspected to make sure they are circumferentially full thickness, including mucosa, and that the esophageal staple line is present in the specimen. Anesthesia will insert a nasogastric tube that can easily be seen passing through the anastomosis. By grasping the tip of the conduit, an absorbable stitch is placed between the esophageal stump and the stomach on the site where the esophageal stump's linear staple line and circular staple line cross. Some omentum can also be secured at this site to protect the anastomosis. Another stay suture is placed between the 


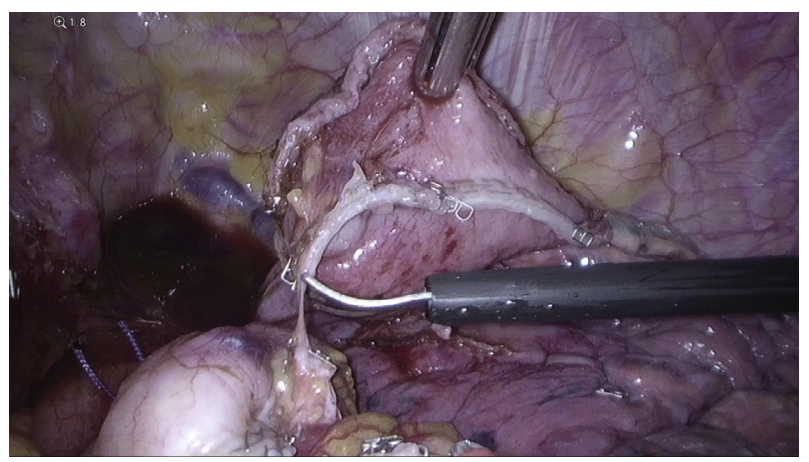

VIDEO 10. Step 10. Video available at: https://www.jtcvs.org/article/ S2666-2507(21)00515-0/fulltext.

esophageal stump and the most proximal tip of the conduit. The anastomosis should be tension free. A linear stapler is used to transect the open end of the proximal conduit, ensuring that at least 1 to $2 \mathrm{~cm}$ of stomach is left between this and the circular staple line of the anastomosis, to avoid ischemia. The stomach is then secured using absorbable tacking sutures to the proximal mediastinal pleura, which helps release tension on the anastomosis and provides pleural coverage to help contain a potential leak. The greater curvature's omentum should be laid on the tracheal side of the anastomosis in order to protect the airway in case of a leak. Any redundant conduit should be gently pushed down into the abdomen, and the staple line should be checked to ensure appropriate orientation of the conduit (Video 10).

\section{Postoperative Course}

Our patients are managed according to a specific early recovery after surgery (ERAS) protocol. Extubation takes place in the operating room. The nasogastric tube remains on suction for two days, and the patient is nil per os to help prevent distension of the gastric conduit and tension on the new anastomosis immediately after surgery. On postoperative day (POD) 2, tube feeds are started and slowly advanced per protocol. The nasogastric tube is removed by POD 3 to 4 , unless output is abnormal or there is significant conduit distension on the radiograph. The chest tube is also removed on POD 3 to 4 unless a chyle leak is present. Diuresis starts on POD 3 and continues while clinically indicated, usually until discharge. A clear liquid diet is started on POD 5. Discharge typically takes place on POD 7. Diet is advanced in the outpatient setting, along with a wean from tube feeds. The jejunostomy is typically removed at the 3-week follow-up visit.

\section{CONCLUSIONS}

Minimally invasive Ivor Lewis esophagectomy is a technically demanding operation requiring careful dissection with attention to detail and purposeful care after surgery.

\section{References}

1. Biere SS, Maas KW, Bonavina L, Garcia JR, van Berge Henegouwen MI, Rosman C, et al. Traditional invasive vs minimally invasive esophagectomy: a multi-center, randomized trial (TIME trial). BMC Surg. 2011;11:2.

2. Luketich JD, Pennathur A, Awais O, Levy RM, Keeley S, Shende M, et al. Outcomes after minimally invasive esophagectomy: review of over 1000 patients. Ann Surg. 2012;256:95-103.

3. Sgourakis G, Gockel I, Radtke A, Musholt TJ, Timm S, Rink A, et al. Minimally invasive versus open esophagectomy: meta-analysis of outcomes. Dig Dis Sci. 2010;55:3031-40.

4. Dantoc M, Cox MR, Eslick GD. Evidence to support use of minimally invasive esophagectomy for esophageal cancer: a meta-analysis. Arch Surg. 2012;147: 768-76.

5. Singh RK, Pham T, Diggs B, Perkins S, Hunter JG. Minimally invasive esophagectomy provides equivalent oncologic outcomes to open esophagectomy for locally advanced (stage II or III) esophageal carcinoma. Arch Surg. 2011;146:711-4.

6. Noble T, Tan KS, Barbetta A, Adusumilli P, Bains M, Bott M, et al. Does pyloric drainage have a role in the era of minimally invasive esophagectomy? Surg Endosc. 2019;33:3218-27. 